\title{
AUDIT ON IN-HOSPITAL PRESCRIBING IN PARKINSON'S
} DISEASE

Michela Simoni, Hannah Martin, Lucy Strens. University Hospital of Coventry and Warwickshire

10.1136/jnnp-2014-309236.19

Background and Objectives Omitting, incorrectly prescribing, or suddenly stopping dopaminergic medications may have significant sequelae. We aimed to assess how accurately PD medications are prescribed on admission to University Hospital of Coventry and Warwickshire (UHCW).

Methods The 2006 Parkinson Disease NICE guidelines were set as standard. Notes and drug charts of PD patients admitted between January and June 2013 were reviewed for possible mistakes in name, dosage, formulation and timing, for omissions and for prescription of dopamine-blocking drugs.

Results Of 296 PD patients with at least one overnight stay, 67 (mean age $79.1+/-6.9$ years, 67\% male) were audited. $21 \%$ of patients had some incorrect prescription (name, formulation, dose, timing) on their clerking notes, and 51\% had a drug chart discordant from their usual regime (mainly timing and formulation errors). $47 \%$ of the audited patients missed at least one dose of their PD medications during admission, and 8 patients (9.9\%) had dopamine-blocking drugs prescribed.

Conclusions Awareness of the importance of correct PD medications prescribing must increase among admitting doctors. Guidelines have been introduced in July 2013 on our Trust intranet, and they have been advertised at grand-rounds. The audit will be repeated at 12 months from their introduction. 